\title{
Improvement of Light Transmission using Photonic Lattices for Solar Cells
}

\author{
Xiaomin Jin and Simeon Trieu \\ Electrical Engineering Department, California Polytechnic State University, San Luis Obispo, CA 93407 \\ xjin@calpoly.edu.
}

\begin{abstract}
We study solar-cell interfaces designs using photonic lattices. We simulate rectangular and triangular micro-profiles as the solar cell surface. Compared to the conventional flat surface, the micro-profile interface can increase light transmission to $98 \%$. OCIS codes: (040.6070); (350.4238)
\end{abstract}

\section{Introduction}

In general, solar cells are very critical for the energy conversion for our future and they are irreplaceable energy source as well. How to increase the efficiency of a solar cell is always an important research topic up to now. The proposed ideas are using cascade solar cells [1], design heat exchanger method (HEM) multi-crystalline solar cells [2, 3], using laser-grooved backside contact [4], simulation results on shaped external quantum efficiency (EQE) [5], and simulation on micromorph solar cell structure[6].

Modeling of solar cells plays an important role for optimization of the device structure and the evaluation of material parameters and designs. In the project, we focus our study on device surface structure design that will enhance light extraction and investigate on the effects that influence the light power transmission efficiency. We first study on application of nano-photonic structure (photonic lattice or photonic crystal) in solar cell design. Further more, we evaluate and compare the various triangular interface structures, and optimize the micro-profiled layer design.

\section{Photonic Lattice Simulation Results and Discussions}

We propose to design a micro-scale photonic grating at the interface of solar cell to enhance the light transmission in order to improve the total absorption of the device, and perform a simulation of diffraction based on simplified two-dimensional (2D) rigorous coupled wave analysis (RCWA) to evaluate the concept of the diffraction grating application. RCWA is a rigorous grating diffraction theory which is used to study the mechanism of the diffraction of light from the periodic structured surfaces [79]. RCWA represents the electromagnetic fields as a sum over coupled waves. Full vector Maxwell's equations are solved in Fourier domain to obtain each coupled wave, which is related to Fourier harmonic. Fourier harmonics are used for the periodic permittivity function in the calculation. Then the diffraction efficiencies are calculated. For each incident angle $\theta$, we calculate the transmitted -20 to +20 order diffraction efficiency. At the end of calculation, we obtained the transmittance by summing all the diffraction efficiencies.

The schematic diagrams of the simulation model which have three kinds of interfaces are shown in Fig. 1. The transmittance diffraction characteristics of the flat interface, rectangular photonic lattice grating, and triangular photonic lattice gratings are analyzed and compared. First, we assume that the gratings period $\Lambda=6 \mu \mathrm{m}$ and the gratings groove depth $\mathrm{d}=3 \mu \mathrm{m}$ are same in Fig. $1(\mathrm{~b})$ and (c). While the bottom width of triangular grating is $\mathrm{w}=3 \mu \mathrm{m}$. In this case, the rectangular-profile is actually a squareprofile. For simplicity in this simulation, we consider lossless dielectrics and incident electromagnetic plane wave polarization perpendicular to the plane of incidence. The results of the three-profile comparison are shown in Fig. 2(a): the transmittance of flat surface (straight line), rectangular profile surface (short dashed line), and triangular profile surface (long dashed line) as function of incident light wavelength. It is very clear that the triangular photonic lattice structure has the highest light transmittance, which is about $75 \%-85 \%$ distributed across the wavelength span up to $1.2 \mu \mathrm{m}$. The squareprofile photonic lattice is also better than the flat surface in general. However its total transmission is oscillating versus the wavelength. And at some wavelength such as $0.6-0.7 \mu \mathrm{m}$, the transmission rate of square-profile is lower than that of the flat surface. Our simulation shows clearly that a partial of blocked light can be extracted by using the transmitted diffraction of the micro-scale photonic-lattice. 


\section{STuC5.pdf}

We also performance simulations for the above three cases at different incident angles, which is shown in Fig. 2(b). The incident angle $\theta$ (as shown in Fig. 1) upon the normal of the grating from a plane wave are varied from 0 to $90^{\circ}$. The calculation is at $700 \mathrm{~nm}$ wavelength. The periodic gratings produce both forward diffracted (transmitted diffraction) and backward diffracted (reflected diffraction) waves. For a given incident angle $\theta$, the transmittance is the sum of the transmitted diffraction efficiency of all orders, which enters into Si through the gratings from the air. It also shows that the triangular-profile photonic lattice has the highest transmission rate at any launch angle.

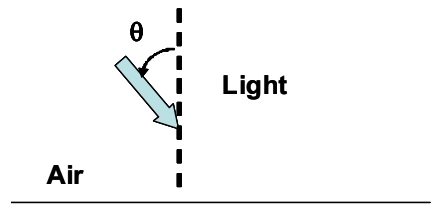

Si

(a)

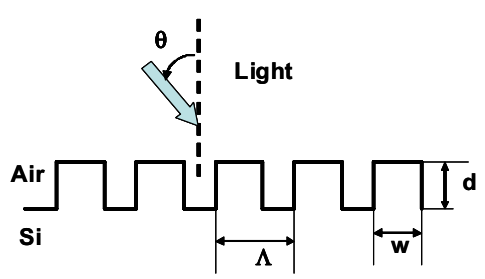

(b)

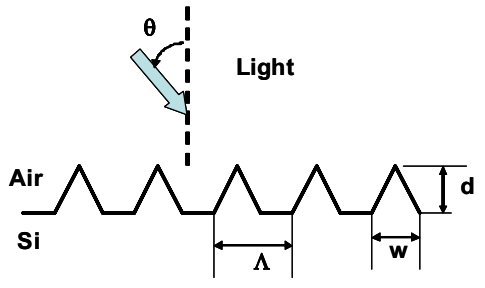

(c)

Fig. 1 The schematic diagrams of solar micro-profiles for the simulation. a) flat interface; b) rectangular interface; and c) triangular interface

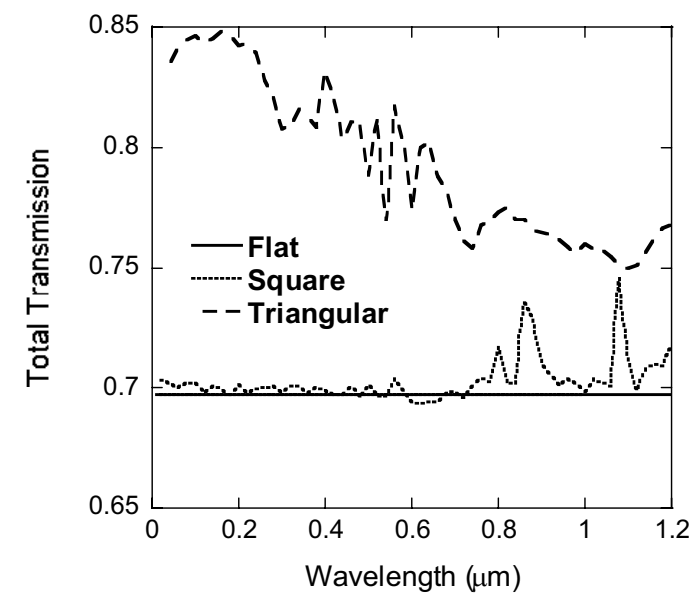

(a)

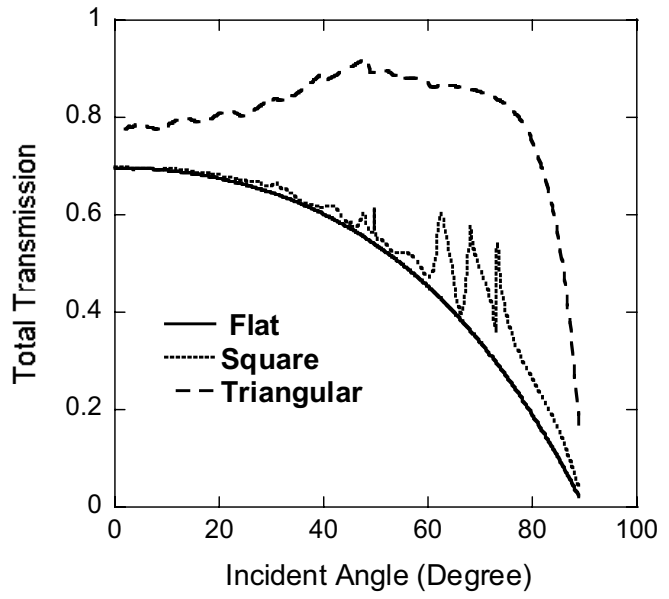

(b)

Fig. 2 The transmittance of flat surface (straight line), rectangular profile surface (short dashed line), and triangular profile surface (long dashed line) as function of (a) incident light wavelength and (b) incident light angle.

Since the triangular-profile photonic lattice has the best performance, we vary the depth $\mathrm{d}$ and width $\mathrm{w}$ to find the optimize design point for this interface structure. Fig. 3 (a) shows the light transmission of $\mathrm{d}=1,2,3,4$, and $10 \mu \mathrm{m}, \mathrm{w}=3 \mu \mathrm{m}$, and $\Lambda=6 \mu \mathrm{m}$. The $\mathrm{w}=3 \mu \mathrm{m}$ and $\mathrm{d}=2 \mu \mathrm{m}$ case has the highest transmission efficiency. Fig. 3 (b) shows the light transmission of $d=3 \mu \mathrm{m}, \mathrm{w}=1,2,3,4,5$, and $6 \mu \mathrm{m}$, and $\Lambda=6 \mu \mathrm{m}$. The largest width $\mathrm{w}=6 \mu \mathrm{m}$ case has the best transmission. From above data, we further simulate $\mathrm{w}=6 \mu \mathrm{m}$ case with $\mathrm{d}=1,2,3,4$, and $10 \mu \mathrm{m}$ (shown in Fig. 4(a)) and other $\mathrm{w}$ and $\mathrm{d}$ combinations. For the case $\mathrm{w}=6 \mu \mathrm{m}$ and $\mathrm{d}=10 \mu \mathrm{m}$, the transmission is above 0.98 from 0.05 to $1.2 \mu \mathrm{m}$ wavelength, which is our best result. Its transmission versus the launch angle at $700 \mathrm{~nm}$ wavelength is shown in Fig. 4(b). Compared to the conventional flat surface, the triangular-profile interface has about $15 \%-30 \%$ improvement of light transmission coefficient, from $70 \%$ (flat surface) to $98 \%$ (triangular surface).

In conclusion, the simulation gives us a clear insight of the transmitted diffraction mechanism for designing the optical interface of solar cells. The diffraction of a well designed grating increases the light transmission and allows a portion of reflected light (of the flat interface) to pass through. If we further optimize the parameters of grating with different periods (even to nano-scale), other profiles, and suitable refractive index of coating, we can improve light extraction efficiency of solar cells. In this simulation, we achieve about $98 \%$ light transmission rate compared to the flat surface.

This work was sponsored by the Department of the Navy, Office of Naval Research, under Award \# N00014-07-1-1152. 


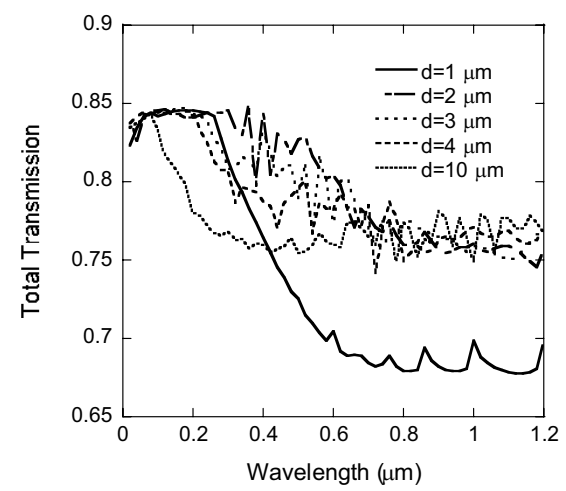

(a)

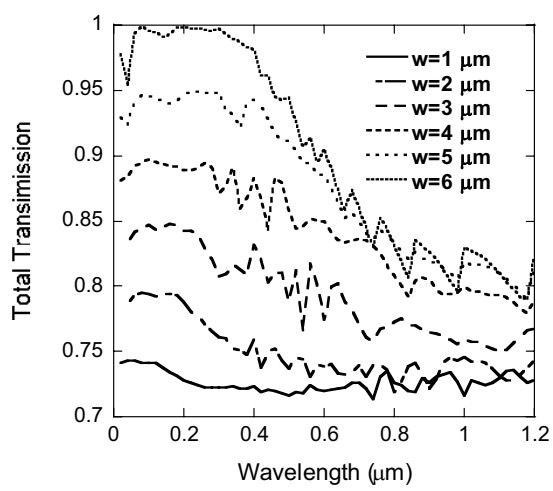

(b)

Fig. 3 The transmittance of triangular-profile interface: (a) $d=1,2,3,4$, and $10 \mu \mathrm{m}, w=3 \mu \mathrm{m}$, and $\Lambda=6 \mu \mathrm{m}$ and (b) $d=3 \mu \mathrm{m}$, w= $1,2,3,4,5$, and $6 \mu \mathrm{m}$, and $\Lambda=6 \mu \mathrm{m}$.

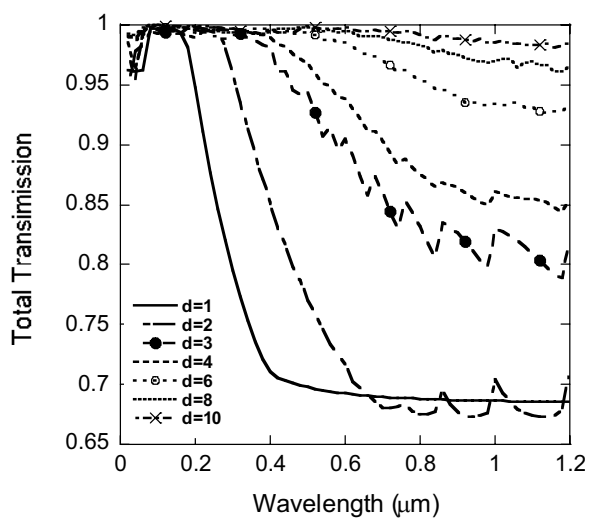

(a)

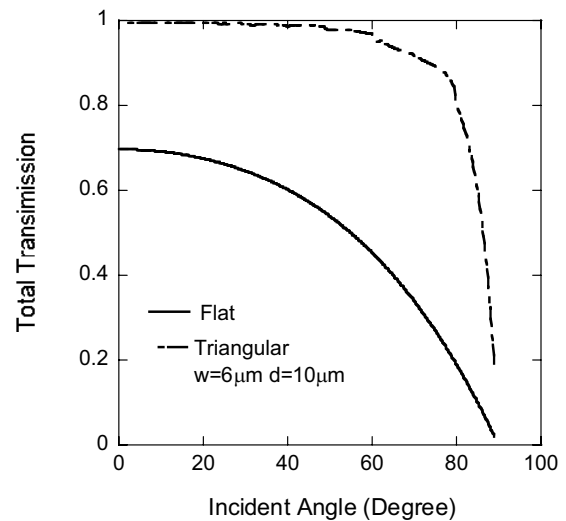

(b)

Fig. 4 The transmittance of (a) triangular-profile interface: $\mathrm{d}=1,2,3,4,6,8$ and $10 \mu \mathrm{m}, \mathrm{w}=6 \mu \mathrm{m}$, and $\Lambda=6 \mu \mathrm{m}$ and (b) triangularprofile interface $\mathrm{d}=10 \mu \mathrm{m}, \mathrm{w}=6 \mu \mathrm{m}$, and $\Lambda=6 \mu \mathrm{m}$ comparison to flat interface

\section{Reference}

[1] F. D. Ho and T. D. Morgan, "SPICE modeling of cascade solar cells," in IEEE Proceedings of Southeastcon 1991, vol.2, 7-10 April 1991, pp.776 - 780 .

[2] A. Rohatgi, S. Narasimha, S. Kamra, P. Doshi, C. P. Khattak,K. Emery, and H. Field, "Record high 18.6\% efficient solar cell on HEM multicrystalline material," Photovoltaic Specialists Conference 1996, Conference Record of the Twenty Fifth IEEE, 13-17 May 1996, pp. $741-744$

[3] H. Lautenschlager, F. Lutz, C. Schetter, U. Schubert, and R. Schindler, "MC-silicon solar cells with $>17 \%$ efficiency," Photovoltaic Specialists Conference 1997, Conference Record of the Twenty-Sixth IEEE, 29 Sept.-3 Oct. 1997, pp.7 - 12.

[4] Jiun-Hua Guo and J. E. Cotter, "Laser-grooved backside contact solar cells with $680-\mathrm{mV}$ open-circuit voltage," IEEE Transactions on Electron Devices, 51, 2186 (2004).

[5] G. Letay, M. Breselge, A. W. Bett, "Calculating the generation function of III-V solar cells," in Proceedings of 3rd World Conference on Photovoltaic Energy Conversion, 2003, Vol 1, 11-18 May 2003, pp741 - 744.

[6] B. E. Pieters, J. Krc, and M. Zeman, "Advanced Numerical Simulation Tool for Solar Cells - ASA5," Photovoltaic Energy Conversion, Conference Record of the 2006 IEEE 4th World Conference on, Vol 2, May 2006, pp.1513 - 1516.

[7] M. G. Moharam, T. K. Gaylord, "Rigorous coupled-wave analysis of metallic surface-relief gratings," J. Opt. Soc. Am. A 3 , 1780 (1986).

[8] Lifeng Li, "New formulation of the Fourier modal method for crossed surface-relief gratings," J. Opt. Soc. Am. A 14, 2758 (1997).

[9] Mingming Jiang, Theodor Tamir, and Shuzhang Zhang, "Modal theory of diffraction by multilayered gratings containing dielectric and metallic components," J. Opt. Soc. Am. A 18, 807 (2001). 